\title{
ANT COLONY OPTIMIZATION FOR SCHEDULING OF PCBS USING SINGLE MACHINE
}

\begin{tabular}{|l|l|l|l|}
\hline Akshaye Malhotra & Vijay Pandey & S.K. Sahana & Somak Datta \\
Department of Production & Department of Production & Department of Production & Department of Production \\
Engineering & Engineering & Engineering & Engineering \\
Birla Institute of Technology, & Birla Institute of Technology, & Birla Institute of Technology, & Birla Institute of Technology, \\
Mesra, Ranchi, India & Mesra, Ranchi, India & Mesra, Ranchi, India & Mesra, Ranchi, India \\
\hline
\end{tabular}

\begin{abstract}
In this paper, problem for scheduling 'N' type of printed circuit boards (PCBs) using single machine assembled with an automatic component interchange mechanism is considered in which the total number of different components required to process ' $\mathrm{N}$ ' PCBs exceeds the capacity of the feeder rack. The objective of this paper is to minimize the total number of component switches. A switch of component type occurs when a component type is removed from the feeder rack and a different component is inserted. With an aim to provide a near optimal solution, the order in which the PCBs are scheduled on the axial insertion machine and the components to be placed on the feeder rack is computed before the processing of the PCBs. Scheduling of individual PCB types on the placement machine and feeder rack minimizes the mean flow time of the assembly. The focus of the research will be on minimizing the mean flow time of all the PCBs' and implicitly minimizes the work-in-process inventories. This paper deals with the scheduling problem in the PCB assembly by using ant colony optimization (ACO) technique.
\end{abstract}

\section{Keywords - PCB, Scheduling, Grouping, Ant Colony Optimization.}

\section{INTRODUCTION}

Semiconductor industry has become one of the largest industries of the world. In this industry, working environment is considered as a very tedious job shop, that involves large and changing varieties of products, sequence dependent setup times, multiple types of work-center's in a dynamic scheduling environment.

Printed-circuit-board (PCB) assembly involves basically two steps, insertion and soldering of electrical components into the printed circuit boards. The modern placement machines are highly expensive, having a price range of US $\$ 300,000$ to $\$ 1,000,000$. Assembly shop requires many machines so the initial investment is required to setup an automated assembly plant is very high and requires proper level of utilization in order to minimize the operating cost.
Following are the steps involved in PCB assembly as shown in Figure 1:

- Printing of circuit on a clad board (laminated copper board).

- Etching.

- Drilling.

- Placing electronic component on board.

- Soldering of component (tin-lead allow).

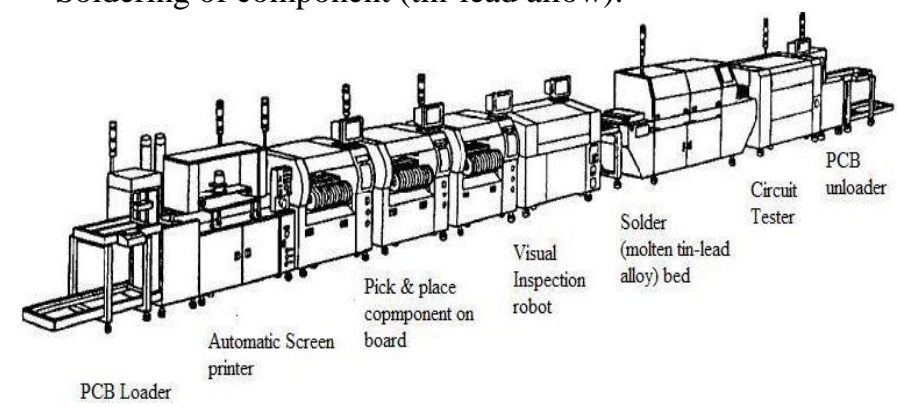

Figure.1. Typical PCB assembly line.

\section{Group Technology}

It is a manufacturing technique in which the elements having the similarities in the manufacturing process are assembled together. GT is very advantageous for engineering, manufacturing and process planning. The similarity between two PCBs' can be calculated by the Jaccard's similarity formula.

$$
\text { Jaccard's similarity between two PCB's }=\frac{\left|P C B_{i} \cap P C B_{j}\right|}{\left|P C B_{i} \cup P S C_{j}\right|}
$$

\section{Ant Colony Optimization technique}

Ant colony optimization is a metaheuristic in which a colony of artificial ants cooperate in finding good solutions to difficult discrete optimization problems. An artificial ant in $\mathrm{ACO}$ is a stochastic constructive procedure that incrementally builds a solution by adding opportunely defined solution components to a partial solution under construction. Therefore, the ACO metaheuristic can be applied to any combinatorial optimization problem for which a constructive heuristic can be defined. [1]. 
An ACO algorithm can be constructed by following these three procedures:

- Construct ants' solution.

- Update pheromones

- Daemon actions

\section{Advantages of ACO:}

- ACO is more applicable for problems where source and destination is predefined and specific.

- It has advantage of distributed computing.

- Positive Feedback accounts for rapid discovery of good solutions.

- Efficient for Traveling Salesman Problem and similar problems.

\section{Rules for updating the pheromone trails}

Rule 1st.

Probability of transition of a virtual ant from node ' $i$ ' to node ' $j$ ' is given by:

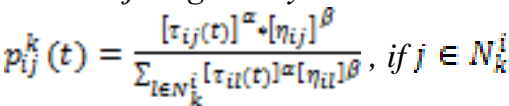

Where, $\eta_{\mathrm{ij}}=\frac{1}{\mathbb{d}_{\mathrm{ij}}}=$ heuristic information

$N_{k}^{i}=$ Feasible neighborhood of ant $k$

$\tau_{i j}=$ Amount of pheromone on the path

$\alpha_{s} \beta=$ Algorithm parameters

Rule 2nd.

Local pheromone $\quad$ updating
$\tau_{i j}(t+1)=(1-\rho) \tau_{i j}(t)+\rho \tau_{0}$

Where, $\rho=$ evaporation factor

Rule 3rd.

Global pheromone updating rule: $\tau_{i j}(t+1)=(1-\rho) \tau_{i j}(t)+\rho \Delta \tau_{i j}(t)$

Where,

$\Delta \tau_{i j}=\frac{Q}{L_{k}}\left(L_{k}=\right.$ length of the $k^{\text {th }}$ ant travel $)$

\section{Overview of the PCB Assembly}

The PCB assembly task consists of placing a number of pre specified electronic components types at the pre specified locations on a PCB. The sequential placement machine, such as the pick-and-place (PAP) machine considered in this study, performs the pick-up and placement operations one-by-one. The configuration of the pick and place machine used in this paper consists of three components: a table to which the PCB is attached, a feeder rack that holds components, and a head that picks components from the feeder and places them on the PCB. A complete board cycle is assumed in which the head starts from a given home position, moves between feeders (on the machine) and placement locations (on the PCB) until all the components have been mounted. The head returns to the home position once all the components required for a board have been placed on the board.

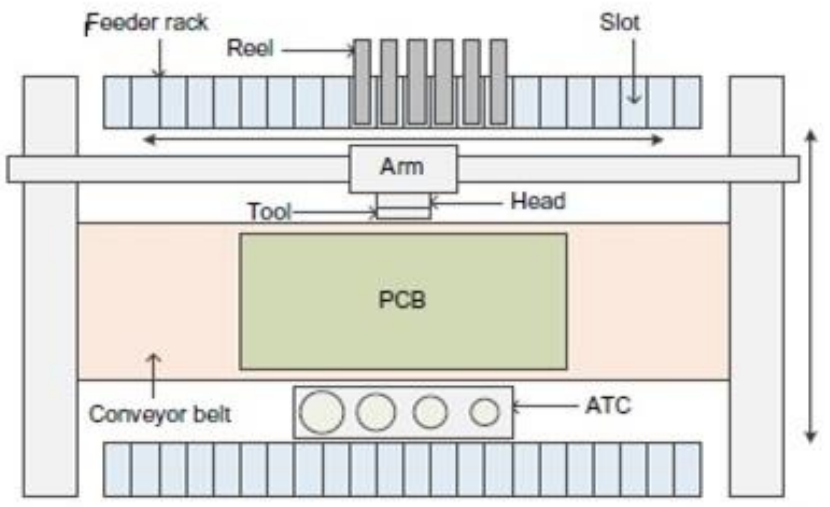

Figure.2. Pick and place assembly machine.

The PCB to be assembled is transferred through the machine on a conveyor belt. There may be one or more feeder racks aside the conveyor belt, which have a certain number of available slots. Components are contained in the reels, which are going to be placed on the PCBs' and each of them contains only one component type: resistors, capacitors, transistors, and integrated circuits, among others. Each slot contains only one reel, while reels may occupy one or more contiguous slots. The machine has a robotic arm, which has one or more heads. Heads pick up components from the reels on the feeder racks and place them on the PCB, by means of using an appropriate tool. Each component type can be picked up with a subset of tools, that is, one head with a specific tool can only pick up components from a limited set of component types. Tools are changed in the automatic tool changer (ATC) when the next component cannot be picked up with the current tool.

In order to minimize the total assembly time, when manufacturing multiple PCBs' the following parameters should be minimized:

- The number of times the feeder rack needs to be reconfigured.

- The total number of changes in the reel of feeder rack.

In general, planning of PCB manufacturing process involves the following steps:

- Partitioning entire set of board types into groups that are to be assigned to placement machines, and forming a sequence of these board groups, in which they are assembled. The decision involving sequencing of different groups of PCBs forms the group-sequencing problem.

- For each machine, a feeder rack assignment, that is an assignment of component types and positions on the feeder rack.

- For each machine and board type, determine the component placement sequences. 
The PCBs are grouped so that the total number of component types in a group is minimized, which means that a maximum number of PCBs are accommodated in a group. At each grouping stage the following parameter that counts the number of new components needed to add a PCB to group is evaluated. The PCB that adds the minimum number of new components is chosen. A new group is formed when the feeder rack capacity $\mathrm{C}$ is exceeded.

\section{BACKGROUND}

The earliest work in PCB scheduling was done by Lin. [2] et al. He developed BLISS (Bi-Level Intelligent Scheduling System), using a two-level scheduling approach integrating product-level and board-level scheduling, to schedule jobs for PCB manufacturing systems. Kim. D. [3] et al., considered PCB grouping problem for a surface-mounting device with a number of component feeders and several types of PCBs to be produced. The problem discussed in this paper is how to group the PCBs so that the total set-up time for component feeders is minimized. Carlos A. Brizuela [4] et al. proposed a genetic algorithm that uses a group-based representation with a series of specialized genetic operators. Yongzhong $\mathrm{Wu}$ [5] et al. investigated the multiline scheduling problem in PCB assembly. The results showed that the established model is able to find optimal solutions while satisfying all the practical constraints. However, the computational complexity is shown to be extremely great. O. Z. Maimon [6] et al. presented a genetic algorithm approach to the component switching problem.

\section{MODEL FORMULATION}

\section{For PCB grouping problem}

Following assumptions were made in order to formulate the problem that minimizes the total number of component switches:

- Different component type acquire only one component slot in the feeder rack.

- The total number of different component types required for processing a group of PCBs' is less than the feeder rack capacity.

This problem provides a set of group ' $G$ ' of PCB types in such a way that the number of resulting groups $|G|$ is minimized without exceeding the number of component types ' $S$ ' that a machine can accommodate.

In order to formulate the problem these following notations are used:

Let,

Types of PCBs' to be processed $=\mathrm{N}(1,2,3, \ldots, \mathrm{N})$

Types of components required for processing ' $\mathrm{N}$ ' $\mathrm{PCBs}$ ' $=\mathrm{M}$ $(1,2,3, \ldots, \mathrm{M})$

Number of components in PCB $i=M_{\mathrm{i}} \leq \mathrm{M}$
Where, $\mathrm{M} \geq \mathrm{N}$

Also, these are the following variables used:

Data Input,

$a_{\mathrm{hi}}=1$, if component type $k$ is used in PCB type $i$ 0 , otherwise.

Decision Variable,

$p_{j i}=1$, if PCB type $i$ is assigned to group $j$.

0 , otherwise.

Decision Variable,

$q_{j}=1$, if group $j$ is formed.

0 , otherwise.

$n_{k j}=\left[\frac{1}{M} \sum_{i=1}^{W} p_{i j} a_{k i j}\right]$

Where,

$n_{k j}=1$, if the component type $k$ is used by at least one PCB in group $j$

0 , otherwise.

With these notations, the PCB grouping problem can be modelled as:

$\min _{p_{i j} q_{j} x_{k j}}|G|=\sum_{q=1}^{N} q_{j}$

Subject to,

$\sum_{k=1}^{M} n_{k j} q_{j}=S$ (Number of component types in each group should not exceed the number of available slots)... [3]

$\sum_{j=1}^{\mathbb{W}} p_{i j} q_{j}=1$ (One PCB type must be assigned to only one group).

$p_{i j}, q_{j,}, n_{k j} \in\{0,1\}$. (Integrality constraint

\section{For PCB Group Sequencing Problem}

Now, given a set ' $G$ ' of empty groups.

This problem consists of sequencing the groups in ' $G$ ' such that the total number of feeder changes ' $C$ ' is minimized.

Let,

Decision Variable,

$x_{j 1}=1$, if PCB group $l$ is manufactured after PCB group $j$ 0 , otherwise.

This problem can be modelled as:

$\min _{x_{j l} \in[0,1]} C_{i \mathbb{i}}=\sum_{j \in G} \sum_{l \in G} \sum_{k=1}^{M}\left(n_{k j}-n_{k i}\right)^{2} x_{j l} \ldots \ldots \ldots$.6]

Equation [6] provides the changes in the feeder rack when finishing the manufacturing of PCB group ' $j$ ' and starting with PCB group ' $l$ '. 
Subject to,

$\sum_{l \in G} x_{j 1}=1, \forall j \in G$ (One PCB group must be manufactured right after the group ' $j$ ')

$\sum_{j \in G} x_{j 1}=1_{v} \forall j \in G$ (One PCB group must be manufactured right after group ' $l$ ').

$$
\begin{gathered}
C_{\text {il }}=1 \text {, if PCB group ' } j \text { ' used component type ' } k \text { ' and PCB } \\
\text { group ' } l \text { ' does not or vice versa } \\
0 \text {, otherwise. }
\end{gathered}
$$

\section{GROUPING STRATEGY FOR PCB ASSEMBLY}

\section{Grouping Strategy}

Grouping of PCBs' is done involving the principle of collecting the PCBs' that require similar components for assembly under a single group. For grouping the PCBs' in different groups, we have assumed that once a reel is loaded in the feeder rack then the quantity of the components in the feeder rack is enough to carry out the assembly of all the PCBs'.

Algorithm for PCB Grouping Problem

STEP1.

Determine the staring SEED PCB. SEED is the $P C B$ requiring the maximum number of components (maximum global similarity) for the assembly.

STEP2.

Start with an empty group ' $G$ ' and assign SEED $P C B$ as the first member of the group and load the components in the feeder rack that are required for its assembly.

STEP3.

For finding the next element of the group, find that unallocated PCB having the maximum number of similar components as compared to SEED. Load all the extra components needed to assemble this PCB and proceed until the feeder rack capacity is exceeded.

STEP4.

When the feeder rack capacity is exceeded, make another empty group and repeat steps 2-4.

\section{STEP5.}

Repeat the above steps until either each PCB forms a part of a group or forms a group of its own.

\section{CASE Study}

\section{For grouping strategy}

In order to explain the grouping strategy, a PCB component incidence matrix is used, which is shown in Table 1.

Given, a lot of 12 PCBs' and the number of different types components contained by a PCB is 10 to 17 . Total number of different types of components is 30 and the feeder rack capacity of the pick and place machine is 20 slots [7].

According to the first step of the algorithm, SEED PCB is determined. The PCB with the maximum number of components is selected as SEED. From Table 1. PCB 7 is selected as the SEED. Place this PCB as the first element of the empty group G1. Second element of the group is selected having the maximum number of similar components with the SEED PCB i.e. PCB 5. If another element is placed in the group $\mathrm{G} 1$ then the feeder rack capacity will be exceeded therefore a new empty group G2 is formed. This process continues until all the unallocated PCBs, are placed in

\begin{tabular}{|c|c|c|c|c|c|c|c|c|c|c|c|c|c|}
\hline & & 1 & 2 & 3 & 4 & 5 & 0 & 7 & 8 & 9 & 10 & 11 & 12 \\
\hline Component & 1 & 1 & 0 & 1 & 0 & 1 & 1 & 1 & 0 & 1 & 0 & 0 & 0 \\
\hline No. & 2 & 0 & 0 & 0 & 0 & 0 & 1 & 0 & 0 & 0 & 1 & 0 & 0 \\
\hline & 3 & 1 & 1 & 1 & 1 & 0 & 0 & 1 & 1 & 0 & 0 & 0 & 0 \\
\hline & 4 & 0 & 0 & 1 & 1 & 1 & 0 & 1 & 0 & 1 & 1 & 0 & 0 \\
\hline & 5 & 1 & 0 & 0 & 1 & 1 & 0 & 1 & 0 & 1 & 0 & 1 & 1 \\
\hline & 6 & 1 & 0 & 0 & 0 & 0 & 0 & 0 & 0 & 0 & 1 & 1 & 1 \\
\hline & 7 & 0 & 0 & 1 & 0 & 0 & 1 & 0 & 1 & 0 & 1 & 1 & 0 \\
\hline & 8 & 1 & 1 & 1 & 1 & 1 & 1 & 0 & 0 & 0 & 1 & 0 & 1 \\
\hline & 9 & 1 & 1 & 0 & 0 & 1 & 0 & 1 & 1 & 0 & 0 & 0 & 0 \\
\hline & 10 & 1 & 0 & 0 & 0 & 0 & 0 & 0 & 1 & 1 & 0 & 0 & 1 \\
\hline & 11 & 1 & 0 & 0 & 0 & 0 & 0 & 0 & 1 & 0 & 1 & 1 & 0 \\
\hline & 12 & 0 & 1 & 0 & 0 & 1 & 0 & 1 & 1 & 0 & 1 & 0 & 1 \\
\hline & 13 & 0 & 1 & 0 & 0 & 0 & 1 & 1 & 0 & 0 & 0 & 1 & 1 \\
\hline & 14 & 0 & 0 & 1 & 1 & 0 & 0 & 0 & 0 & 1 & 0 & 0 & 0 \\
\hline & 15 & 1 & 0 & 0 & 1 & 1 & 0 & 0 & 0 & 0 & 1 & 1 & 1 \\
\hline & 16 & 1 & 0 & 0 & 0 & 1 & 0 & 1 & 1 & 0 & 1 & 0 & 0 \\
\hline & 17 & 0 & 0 & 1 & 1 & 1 & 0 & 1 & 0 & 0 & 0 & 0 & 1 \\
\hline & 18 & 0 & 0 & 0 & 0 & 0 & 0 & 1 & 0 & 0 & 0 & 0 & 0 \\
\hline & 19 & 0 & 0 & 0 & 0 & 0 & 1 & 0 & 0 & 0 & 0 & 0 & 0 \\
\hline & 20 & 0 & 0 & 0 & 1 & 0 & 1 & 1 & 0 & 1 & 0 & 0 & 1 \\
\hline & 21 & 0 & 1 & 0 & 1 & 0 & 0 & 0 & 0 & 0 & 1 & 0 & 0 \\
\hline & 22 & 1 & 1 & 0 & 0 & 0 & 1 & 1 & 0 & 0 & 0 & 1 & 1 \\
\hline & 23 & 0 & 1 & 0 & 0 & 0 & 0 & 0 & 1 & 0 & 1 & 1 & 0 \\
\hline & 24 & 0 & 0 & 0 & 0 & 0 & 0 & 1 & 1 & 1 & 0 & 1 & 1 \\
\hline & 25 & 1 & 0 & 1 & 0 & 1 & 0 & 1 & 1 & 1 & 0 & 1 & 1 \\
\hline & 26 & 0 & 1 & 0 & 1 & 0 & 1 & 1 & 0 & 1 & 0 & 0 & 1 \\
\hline & 27 & 0 & 0 & 1 & 0 & 0 & 1 & 0 & 0 & 0 & 0 & 0 & 0 \\
\hline & 28 & 0 & 1 & 1 & 1 & 0 & 0 & 1 & 0 & 1 & 1 & 0 & 0 \\
\hline & 29 & 1 & 1 & 1 & 1 & 0 & 1 & 0 & 1 & 1 & 0 & 0 & 0 \\
\hline & 30 & 0 & 1 & 1 & 1 & 0 & 0 & 1 & 1 & 0 & 1 & 0 & 0 \\
\hline & $\Sigma$ & 13 & 12 & 12 & 13 & 10 & 11 & 17 & 12 & 11 & 13 & 10 & 13 \\
\hline
\end{tabular}
different groups or forms a group of its own.

PCB No. $\rightarrow$

Table 1. Incidence Matrix showing components that are required to assemble the 12 PCBs' and the total number of components required by each $\mathrm{PCB}$.

Following this procedure, 6 groups are formed having elements given below in Table No 2:

\begin{tabular}{|l|l|l|}
\hline Group Number & PCB Number & $\begin{array}{l}\text { Component Types } \\
\text { Req. } \\
\mathrm{S} \leq 20\end{array}$ \\
\hline G1 & 7,5 & 19 \\
\hline
\end{tabular}




\begin{tabular}{|l|l|l|}
\hline G2 & 12,11 & 16 \\
\hline G3 & 1,8 & 18 \\
\hline G4 & 4,9 & 17 \\
\hline G5 & 10,2 & 19 \\
\hline G6 & 3,6 & 18 \\
\hline
\end{tabular}

Table 2. Shows the number of PCBs' assigned to different groups and component types required to assemble these groups. ( $\mathrm{S}=$ Feeder rack capacity)

Now, all the PCBs are assigned to different groups, (as shown in Table 2) we have to sequence these groups in such a way that the total number of component switches becomes minimum. For this purpose, an algorithm is designed to minimize the number of feeder rack changes using Ant Colony Optimization (ACO) technique.

The 6 groups formed must be sequenced in order to minimize the setup time. Table 3 shows the different component types required to assemble each group.

23
24
25
26
27
28
29
30
$\sum$

Table 3. Incidence Matrix showing components that are required to assemble the 6 groups and the total number of components required by each group.

Algorithm for solving PCB group sequencing problem using ACO

STEP1. Set the different parameters and initialize the pheromone trails.

STEP2. Calculate the number of similar elements in each group.

STEP3. Start iteration 1. Each ant is positioned at the starting node according to the distribution strategy (each node has at least one ant).

STEP4. For $k=1$ to $m$ ( $k=$ number of ants, $m=$ number of nodes), move each ant to different route and repeat until all nodes are visited once.

STEP5. Compute candidate list according to the heuristic information.

STEP6. Select the node to be visited next (from candidate list).

STEP7. A local pheromone updating rule is applied until ant $k$ has completed the tour.

STEP8. When all the ants have completed their tour, pheromone is updated according to the global updating rule also, considering the evaporation of pheromone.

STEP9. Update the heuristic parameters until end condition is met or an optimal solution is obtained.

Above algorithm provides the optimal solution to the sequencing problem and gives the sequence which has minimum number of component switches.

Group sequence with minimum number of component switches is given as:

$G 6 \rightarrow G 1 \rightarrow G 4 \rightarrow G 3 \rightarrow G 5 \rightarrow G 2$

\section{CONCLUSION AND FUTURE SCOPE}

In this paper, problem for scheduling ' $\mathrm{N}$ ' type of printed circuit boards (PCBs) using single machine is discussed. The present research work presents ACO algorithm approach to minimize 
the number of component switches. Algorithm computes the solution in relatively very small time.

Results can be summarized as:

- The developed model describes the characteristics of the complex PCB assembly and its scheduling.

- Model developed is capable of solving highly complex industry-size PCB scheduling problems quickly.

- ACO technique provides the solution to the industrial scheduling problem within seconds.

The described problem can be extended to multi machine environment where more constraints can be taken to solve the problem.

\section{REFERENCES}

[1] M. Dorigo, “Ant Colony Optimization”, 1992.

[2] Lin, F. R., Locascio, A., and Shaw, M. J., "Scheduling Printed Circuit Board Production Systems Using the TwoLevel Scheduling Approach", Journal of Manufacturing Systems, Vol. 16, No. 2, 1997, pp.129 -149.

[3] Yu, S., Kim D., And Park S., "Integer programming approach to the printed circuit board grouping problem", International Journal of Production Research, Vol. 43, No. 8, 2005, pp.1667 -1684.

[4] Carlos A. Brizuela. (2014), "An efficient genetic algorithm for setup time minimization in PCB assembly", Int J Adv Manuf Technology.

[5] Yongzhong Wu. (2009), “A scheduling problem for PCB assembly: a case with multiple lines", Int J Adv Manuf Technol (2009) 43:1189-1201.

[6] O. Z. Maimon (1998), "A genetic algorithm approach to scheduling PCBs on a single machine", Int. J. Prod. Res.,

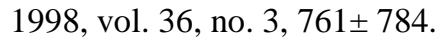

[7] R. Narayanswami, V. Iyengar (2005), "Setup reduction in printed circuit board assembly by efficient sequencing", Int J Adv Manuf Technol (2005) 26: 276-284 